\title{
MedienPädagogik
}

Zeitschrift für Theorie und Praxis der Medienbildung

www.medienpaed.com

\section{Digitalisierung in der Hochschullehre}

\section{Erfahrungen mit dem MathEdu Digital-Lehrkonzept und zur Akzeptanz digitaler Lehrelemente durch die Studierenden}

\author{
Astrid Beckmann
}

\begin{abstract}
Zusammenfassung
Für die Digitalisierung in der Hochschullehre stehen viele verschiedene digitale Möglichkeiten, Ansätze und Tools zur Verfügung. Auf Basis der Forschungsliteratur werden zusammengefasst fünf Ansätze identifiziert, nämlich: Mobile Technologien, Online-Kursprogramme, Learning Objects und Digital Libraries (Repositorien), Technologische Umgebungen für kollaboratives Lernen und Digitalisierte Lehrkonzepte. Im Projekt MathEdu Digital der PH Schwäbisch Gmünd werden die digitalen Ansätze und Tools kritisch analysiert und im Rahmen eines selbst entwickelten Lehrkonzepts erprobt, zunächst erfahrungsbasiert und über Studierendenbefragungen, später systematisch empirisch. Ziel ist es, die mathematikdidaktische Lehre geeignet so anzulegen, dass die Lehramtsstudierenden vielseitige digitale Erfahrungen sammeln können. Gleichzeitig geht es um die Identifizierung und Beseitigung von Barrieren für (auch weniger digital erfahrene) Lehrende. Als Ergebnis zeichnet sich ab, dass als Grundkonzept das Blended-Learning-Format mit digitalen und nicht-digitalen Elementen und der Einsatz eines Learning-Management-Systems mit eingepflegter Digital Library und Learning Objects als geeignet einzustufen sind. VideoDemos sind sehr zu empfehlen, aber auch Webinare, die mit ihren verschiedenen integrierten Funktionen insbesondere auch die Kommunikation und Interaktion unterstützen. Für die unkomplizierte Webinarplanung hat sich ein «Regieplan», eine vorbereitete Tabelle, bewährt, der diesem Beitrag anhängt ist.
\end{abstract}

Digitization in University Teaching - Experiences with the MathEdu Digital Teaching Concept and Students' Acceptance of Digital Teaching Elements

When it comes to the digitization of university teaching, there is no shortage of available options, approaches, and tools. Based on the research literature a total of five different approaches is identified: mobile technologies, online course programs, learning objects and digital libraries (repositories), technological environments for collaborative learning, and digitized teaching concepts. In the MathEdu Digital project at the University of Education Schwäbisch Gmünd, Germany, digital approaches and tools are subject to critical analysis and testing as part of a specially developed teaching concept, which is based on experiences and student surveys initially before becoming methodical and empirical. The objective is to structure the math-didactical teaching so that the 
students gain versatile digital experiences, while at the same time, there's a focus on the identification and elimination of barriers for university teachers also/especially for those of having few digital experience. Based on the findings, as a basic concept, the blended learning format featuring digital and non-digital elements and the use of a learning management system with a well-maintained digital library including learning objects can both be classed as suitable methods. Video demos and webinars also come highly recommended, with the various integrated functions of the latter providing a particular boost to communication and interaction. For a smooth webinar planning, the use of a "direction plan», a prepared spreadsheet, has been successfully proven and tested, which is attached to this paper.

\section{Digitalisierung in der Hochschullehre}

\section{Vorgedanke}

Lehrende an Schulen und Hochschulen und damit speziell auch Lehrende in der Lehramtsbildung übernehmen eine ganz besondere Verantwortung für unsere Zukunft in einer digitalisierten Welt. Die Lehramtsstudierenden sind deshalb derart zu fördern, dass sie erfahren, gebildet, reflektiert und zukunftsorientiert agieren und unterrichten können. Dazu gehört nicht nur, dass sie in die Lage versetzt werden, digitale Medien fachbezogen im Unterricht einzusetzen, sondern sie sollten darüber hinaus auch allgemeine Anwendungen kennen und unkompliziert damit umgehen können. Dies wird durch eigene digitale Erfahrungen unterstützt, die die Lehramtsstudierenden während ihres Studiums etwa in Lehrveranstaltungen sammeln. Hochschullehrende müssen diese Erfahrungen ermöglichen, auch wenn es für sie selbst eine persönliche Herausforderung darstellen kann. Im vorliegenden Beitrag wird von einem Projekt berichtet, das auf ein Lehrkonzept zielt, bei dem Lehramtsstudierende in der mathematikdidaktischen Lehre vielseitige digitale Erfahrungen sammeln sollen und das für - auch weniger digital erfahrene - Lehrende einen möglichst barrierefreien Einsatz digitaler Lehrelemente bieten soll und gleichzeitig offen für die eigene und innovative Weiterentwicklung ist.

\section{Digitale Ansätze und Tools (Lehrelemente) für die Hochschullehre}

Für die Digitalisierung in der Hochschullehre stehen viele verschiedene digitale Möglichkeiten zur Verfügung. Borba et al. (2016) identifizieren in ihrer umfassenden Auswertung internationaler wissenschaftlicher Journale für den Bereich Mathematical Education fünf allgemeine Trends. In eigener Weiterentwicklung, Aktualisierung und Verallgemeinerung dieser Trends ergeben sich zusammengefasst folgende fünf Ansätze für digitalisierte Hochschullehre, Tabelle 1, vgl. auch (Beckmann 2020). 


\begin{tabular}{|c|c|}
\hline Digitaler Ansatz & Beispiele für enthaltene digitale Lehrelemente (Tools) \\
\hline $\begin{array}{l}\text { Mobile Technologien, } \\
\text { i.d.R. für den interaktiven } \\
\text { Gebrauch in Präsenzveran- } \\
\text { staltungen }\end{array}$ & $\begin{array}{l}\text { Tablets z.B. mit fachspezifischer Software, Smartphones, grafikfä- } \\
\text { hige Taschenrechner }\end{array}$ \\
\hline Online-Kursprogramme & $\begin{array}{l}\text { MOOCs (Massive Open Online Courses), geschlossene und ziel- } \\
\text { gruppenorientierte Kursangebote wie Virtual Universities }\end{array}$ \\
\hline $\begin{array}{l}\text { Learning Objects (LOs) } \\
\text { und Digital Libraries, } \\
\text { also Repositorien, in denen } \\
\text { selbst entwickelte oder ver- } \\
\text { breitete LOs zusammenge- } \\
\text { fasst und gespeichert sind }\end{array}$ & $\begin{array}{l}\text { LOs: } \\
\text { - Online-lectures, Lehrvideos, VideoDemo/Audio-Folien-Präsen- } \\
\text { tation (jeweils selbst entwickelt oder von Video-Portalen wie } \\
\text { Youtube usw.), Konferenzvorträge, } \\
\text { - } \quad \text { Apps zur Nutzung bei Aufgabenbearbeitungen, für Games, Vo- } \\
\text { ting, Tests, Umfragen, Social-Media-Protfolios, Bar codes usw. } \\
\text { - Literatur-Dateien, z.B. als pdf, etwa über URLs bereit gestellt } \\
\text { - Software mit Aufgaben und Übungen einschliesslich Simulati- } \\
\text { onen (z.B. online oder auf Tablets angeboten) }\end{array}$ \\
\hline $\begin{array}{l}\text { Technologische Umge- } \\
\text { bungen für kollaboratives } \\
\text { Lernen }\end{array}$ & $\begin{array}{l}\text { LMS - Learning Management Systems wie z.B. Moodle, mit Kom- } \\
\text { ponenten wie } \\
\text { - } \quad \text { Digital libraries } \\
\text { - } \quad \text { Angeboten für kollaborative Arbeit wie Foren, Studierenden- } \\
\text { ordner, E-Mails usw. }\end{array}$ \\
\hline Digitalisierte Lehrkonzepte & $\begin{array}{l}\text { z.B. E-Lectures (also digital angebotene Lehre, insbesondere Lehr- } \\
\text { videos einschliesslich interaktiver digitaler Elemente) und } \\
\text { Blended-Learning-Format, z.B. als Kombination aus } \\
\text { - E-Learning-Angeboten } \\
\text { - Präsenzveranstaltungen } \\
\text { - } \quad \text { Forums-/E-Mail-Austausch } \\
\text { - Webinaren, d.h. online angebotenen Seminaren, die Präsen- } \\
\text { tations- und Interaktionsformate einschliessen wie Video, } \\
\text { Audio, Gruppenraum, Chat, Whiteboard, Votes, Befragungen } \\
\text { usw. }\end{array}$ \\
\hline
\end{tabular}

Tab. 1.: 5 Ansätze für die digitalisierte Hochschullehre.

Eine erfolgreiche digitalisierte Hochschullehre erfordert eine fachspezifisch begründete Entscheidung, welche der Ansätze ausgewählt und wie die Ansätze geeignet kombiniert werden sollen, sowie welche einzelnen digitalen Tools jeweils zu nutzen sind. 
Forschungs- und Entwicklungsstand zu Wirksamkeit und Akzeptanz digitaler Tools in der (mathematikdidaktischen) Hochschullehre

\section{Ein grosses Forschungsdesiderat}

Vor dem Hintergrund einer Bewertung digitaler Tools für die Lehre stellen Trenholm, Alcok und Robinson (2012 und 2015) als Ergebnis ihrer umfassenden Auswertung internationaler wissenschaftlicher Journale fest, dass es kaum empirische Studien zur Digitalisierung in der Lehre gibt. Speziell sehen sie in einer kontextbezogenen Forschung einen grossen Nachholbedarf, die die Unterschiede in den Fachkulturen berücksichtigt. Eine eigene fachbezogene Auswertung der auf den Jahrestagungen der mathematikdidaktischen Community (GDM-Tagungen) präsentierten Forschungen der letzten sieben Jahre bestätigt die Beobachtung des Forschungsdesiderats für den deutschsprachigen Raum, indem es speziell kaum Arbeiten gibt, die sich mit der Digitalisierung in der mathematikdidaktischen Lehre, insbesondere Sekundarstufenlehrämter, befassen. Borba et al. (2016) vermissen in den wissenschaftlichen Arbeiten zudem einen Fokus auf die Interaktion und Zusammenarbeit zwischen Studierenden. Clements, Pawlowski und Manouselis (2015) nennen als ein weiteres Entwicklungsfeld die Qualitätssicherung, vgl. dazu auch (Hahn und Puscher 2017). Trenholm, Alcok und Robinson begründen an verschiedenen Stellen, dass neue und weitere Studien erforderlich sind, etwa, da sie widersprüchliche Befunde in verschiedenen Arbeiten entdecken wie zum Beispiel bei (Feinstein 2010) versus (Owston, Lupshenyuk und Wideman 2011), aber auch, da für eine Beurteilung von digitaler Lehre eine detailliertere Beschreibung der genutzten digitalen Tools erforderlich ist als dies in vielen Publikationen erfolgt. Insgesamt deuten die Ergebnisse und Arbeiten zur Digitalisierung (insbesondere auch auf internationaler Ebene) darauf hin, dass die bisherige Praxis digitalisierter Lehre einige Desiderate und somit einen Entwicklungsbedarf aufweist.

\section{Zur Nutzung digitaler Tools in der Hochschullehre}

Qualitätsgesicherte erfolgreiche digitalisierte Lehre erfordert eine überlegte Herangehensweise, aber auch einen grossen Entwicklungsaufwand - etwas, das Lehrende oft gar nicht alleine oder zusätzlich leisten können. Stichpunktartige Befragungen einzelner Hochschullehrender in Deutschland bestätigen auch, dass Digitalisierung eher (oder überhaupt nur) umgesetzt wird, wenn die Hochschule eine umfassende technische und personelle Unterstützung bereithält. Diesen besonderen Bedarf an einer «unkomplizierten und individuellen Unterstützung der Dozierenden ad hoc und bedarfsorientiert» bestätigt auch die Befragung in (Hetzner et al. 2017, 115). Die Studie von Sailer et al. (2018) an bayerischen Hochschulen zeigt, dass sich nur etwa 
die Hälfte der Lehrenden in der Lage sehen, passive digital gestützte Lernaktivitäten zu planen und umzusetzen und sogar nur etwa jeder Vierte die Fähigkeit zur Gestaltung interaktiver Lernaktivitäten angibt. Jokiaho und May $(2017,20)$ weisen in Bezug auf die Nutzung von E-Learning an Hochschulen auf einen offensichtlichen «Widerspruch zwischen Wunsch und Realität» hin.

Zur Durchführung von E-Lectures (im Vergleich zu Präsenz-Lehrveranstaltungen) fassen Trenholm, Alcok und Robinson (2012 und 2015) in der erwähnten Arbeit zusammen, dass Lehrende die Effektivität und das Nutzen von Routinen schätzen, während die Studierenden die Flexibilität und die Autonomie beim Lernen, die Möglichkeit der Pause und Wiederholung sowie die Bequemlichkeit und gelegentlich auch die klare Strukturiertheit von E-Lectures hervorheben. Als Nachteil empfinden Lehrende, dass sie Emotionen eher schlecht vermitteln können und Studierende vermissen das direkte Feedback. Gadanidis und Borba (2008 bzw. Gadanidis 2012) weisen auf das grosse Potential von Personal Networks (PLN) hin. Trenholm, Alcok und Robinson beschreiben zudem, dass mit der digitalisierten Lehre eine Abnahme der Interaktion einhergehen kann und dass die wenigen empirischen Studien nicht immer einen Erfolg der digitalen Lehre aufzeigen: so schnitten danach in einem Projekt die Studierenden, die E-Lectures ansahen, gegenüber den Studierenden aus Präsenzveranstaltungen schlechter ab, selbst wenn sie immer wieder individuelle Pausen eingelegt haben. Gemäss Le et al. (2010) nutzen Studierende, die nach der surfaceStrategie (oberflächlich, vgl. Cretchly 2005) lernen, häufiger die Pausenfunktion, wobei «diese Funktion von E-Lectures den Studierenden das Erinnern, aber nicht das Verstehen des Materials ermögliche». (Le et al., eigene Übersetzung in Anlehnung an Trenholm et al. 2012, 707).

\section{Kriterien für erfolgreiche digitale Hochschullehre}

Grundsätzlich werden digitalen Tools bereichernde Eigenschaften zugeschrieben, da sie auf ein individualisiertes und lernerzentriertes Lernen ausgerichtet sind (vgl. Downes 2005), diverse Lernstile berücksichtigen, ein eigenes Lerntempo ermöglichen und zu mehr Rückmeldung beitragen (vgl. z.B. Rickard 2010) und insgesamt mehr studentisches Engagement herausfordern können. Mayrberger (2017) weist in diesem Zusammenhang darauf hin, dass nicht die jeweils neuste, digitale Technologie zu einem veränderten Lernen führt, «sondern die Frage der Qualität und Quantität der potentiellen Räume für studentisches Engagement und Partizipation entscheidend sind» (Mayrberger 2017, 20). Verschiedene Forschungen haben gezeigt, dass gerade das eigene Engagement der Studierenden beim Lernen der wichtigste Erfolgsfaktor im Studium ist (Winteler und Forster 2008), wozu auch Feedback und Austausch mit anderen Studierenden und Lehrenden gehören (Mayrberger 2017). Chatti et al. forderten schon vor einigen Jahren ein Umdenken, nämlich weg von 
traditionellen Lernmethoden («knowledge-push») hin zu einer stärkeren Studierendenorientierung («knowledge-pull») mit LOs, die entsprechend sozial, offen und dynamisch sind. Kernelemente der Lehr-/Lernumgebung sollen also sein: Personalisierung, Partizipation, Knowledge-Pull (Chatti et al. 2010).

\section{Zur Akzeptanz digitaler Tools durch Studierende}

Verschiedene Publikationen befassen sich mit der Akzeptanz digitaler Tools. In ihrer umfassenden Auswertung zahlreicher Arbeiten und eigener Untersuchungen entwickeln Venkatesh et al. (insb. 2003, sowie Venkatesh und Zhang 2010) die «Unified Theory of Acceptance and Use of Technology» (UTAUT), in der Akzeptanzkriterien zusammengefasst sind, wie z.B. erfahrene Nützlichkeit, Arbeitserleichterung, Leistungssteigerung, soziale Bedeutung und Einstellung, die je nach Alter, Geschlecht und insbesondere Kultur auch unterschiedlich ausgeprägt sein können. Die UTAUT bezieht sich jedoch auf Arbeitnehmerinnen und Arbeitnehmer in Unternehmen und ist damit nicht direkt auf die Hochschullehre übertragbar. Dazu sind Arbeiten aufschlussreich, die sich mit der Frage befassen, wie Studierende die in der universitären Lehre eingesetzten digitalen Tools bewerten und nutzen. Allerdings muss eingeräumt werden, dass nur in wenigen dieser Arbeiten eine klare Spezifikation der eingesetzten digitalen Lehrelemente erfolgt und auch angegeben wird, ob das Tool ein freiwilliges Add-On oder die Nutzung Pflicht ist und ob es die Präsenzveranstaltung vollständig ersetzt. Eine der Studien, die dies spezifiziert, ist die von Gegenfurter, Fisch und Reitmaier-Krebs (2017): Die nach Abschluss der angebotenen Webinar/ Moodle-Kurse durchgeführten Leitfadeninterviews offenbaren Unterschiede bei der Akzeptanz von Digitalisierung in den Fachkulturen Mathematik und Kindheitspädagogik. In der Studie von Rettig und Warszta (2016), in der die Bewertung der berufsbegleitenden Online-Kurse über eine Vignettenstudie erfolgte, zeigte sich, dass die Kommunikation mit den Lehrenden und deren Feedback, ganz besonders aber die Interaktion der Studierenden untereinander, wesentliche Faktoren für den Erfolg der digitalen Medien sind. Auf die Bedeutung des Feedbacks, aber auch der Sequenzierung der Lerninhalte, weisen auch die in der Arbeit von Frerich und Frye (2016) beschriebenen studentischen Bewertungen des Blended-Learning-Angebots in den Ingenieurwissenschaften hin. Insgesamt scheint die Mischung verschiedener Komponenten förderlich; jedenfalls legt dies die Studie des National Center for Academic Transformation (NCAT) nahe (Rickard 2010). Danach enthält ein erfolgreicher Kurs Online-Tutorials, ist individualisiert und bietet kontinuierliche Betreuung, Feedback, On-Demand-Unterstützung und fördert die Interaktion zwischen den Studierenden. Hahn und Puschner (2017) zeigen, dass Studierende «reine Vorlesungsaufzeichnungen», «statische Kamera» und «Über-Länge» negativ bewerten, und die Arbeit von Makori (2015) mit Post-Graduate Studierenden ergab, dass neben der bereitgestellten 
und gut zugänglichen Infrastruktur zugeschnittene Programme erforderlich sind, die die Studierenden in regulären Lehrveranstaltungen als auch in Social-Media-Plattformen erreichen und anregen. Söbke und Reichelt (2016) und Guo, Goh und Luyt (2017) untersuchen mit Fragebögen und Interviews die Akzeptanz bzw. die Lernwirksamkeit von speziellen Spiel-Apps (QuizUp. bzw. digitales Spiel/Game basiertes Lernen, DGBL). Im Ergebnis zeigte sich, dass die Studierenden die App als Lernwerkzeug sehr unterschiedlich von «überflüssig» über «belebend» bis «prüfungsvorbereitend» bewerteten; bei Guo, Goh und Luyt bewirkte das Spiel zwar Aufmerksamkeit, Zufriedenheit, gefühlsbezogene Freude und erfahrene Nützlichkeit, allerdings gab es keine Hinweise darauf, dass es wirklich Bedeutung für die Studierenden hatte, Vertrauen weckte oder kognitives oder verhaltensbezogenes Vergnügen erzeugte. Dass zumindest einige dieser Indikatoren wichtig für die Akzeptanz von digitalen Medien sind, zeigten Lai und Rushikesh Ulhas (2012) in ihrer Studie zur Akzeptanz von E-Textbüchern mit Universitätsstudierenden, wonach auf Basis ihres Integrierten Modells zur Akzeptanz die erfahrene Nützlichkeit, die Bequemlichkeit, die Vereinbarkeit und die erfahrene Freude wichtige Akzeptanzkriterien für digitale Tools sind.

\section{Projektbeschreibung MathEdu Digital}

\section{Zielsetzung und Fragestellung}

Das Projekt MathEdu Digital der Pädagogischen Hochschule Schwäbisch Gmünd/ Deutschland unter Leitung der Autorin richtet sich zusammengefasst auf folgende zwei Fragenkomplexe (vgl. auch Beckmann 2019a):

- Welche der vielfältigen Digitalisierungsmöglichkeiten eignen sich in der praktischen Umsetzung tatsächlich für die Lehre (hier Mathematikdidaktik der Sekundarstufen) und sind förderlich für die (autonomen oder interaktiven) Lernaktivitäten und digitalen Erfahrungen der Studierenden? Dabei liegt ein Fokus einerseits auf den realen Gelingensbedingungen, Barrieren und Chancen und andererseits auf ausgewählten Aspekten wie Interaktion und Kommunikation und Akzeptanz der digitalen Tools durch die Studierenden.

- Wie gelingt es Lehrende auch mit weniger Affinität zu digitaler Lehre für deren Umsetzung zu interessieren und zu motivieren und sie zu einer möglichst barrierefreien kompetenten und kritischen Auseinandersetzung damit anzuregen?

Im Vordergrund des Projekts steht das Ziel, die mathematikdidaktische Lehre geeignet so anzulegen, dass Studierende vielseitige digitale Erfahrungen sammeln können. Ausgangspunkt ist gemäss (Dehne, Lucke und Schiefner-Rohs 2017, 71f.) also zunächst der Ansatz «Perspektive der Medien», indem nach deren «Potentialen ... als 
Lehr-Lernwerkzeuge», hier in «konkreten Veranstaltungen oder in Eigenverantwortung», gefragt wird. Gleichzeitig geht es um die Identifizierung und Beseitigung von Barrieren für Lehrende. Zum Beispiel weisen auch Jokiaho und May $(2017,28)$ darauf hin, dass es kaum Forschung gibt, die sich mit dem «Abbau von Hindernissen bei der Nutzung von E-Learning» befasst. Entsprechend geht es zunächst um die eigene kritische Befassung, wozu die zielgruppenorientierte Auswahl der digitalen Tools und ihre spezifische Bewertung, insbesondere bezüglich ihrer Eignung und Chancen beim fachlichen Kompetenzerwerb und der Gelingensbedingungen bzw. Barrieren beim Einsatz, gehören. «Digitalisierung in der Lehre» wird vielperspektivisch aufgegriffen, indem sowohl die Lehramtsstudierenden als auch die Lehrenden in den Blick genommen werden, wie auch die Hochschulleitungen. Die drei Bausteine des Projekts sind (1) Inhalte und Medien: hier speziell Mathematikdidaktik und Unterrichtsmedien für den Mathematikunterricht der Sekundarstufen, (2) Digitalisierte Lehre und (3) Digitalisierungspaket, das speziell die Zusammenstellung von bewährten Materialien für Studierende und Lehrende, Hinweise und Anregungen für einen möglichst barrierefreien Einsatz digitaler Tools, auch vor dem Hintergrund einer geeigneten Hochschulstrategie mit gezielten und wirksamen Unterstützungsmassnahmen, beinhaltet. Der Schwerpunkt des vorliegenden Beitrags liegt auf dem Baustein «Digitalisierte Lehre».

\section{Methode}

Das MathEdu Digital-Projekt gliedert sich in zwei methodische Phasen: In der ersten Phase ist das Vorgehen vorwiegend qualitativ und erfahrungsbasiert und zielt zunächst auf die Auswahl geeigneter digitaler Elemente und die Entwicklung und kontinuierliche Verbesserung und Innovierung eines Lehrkonzepts für die konkrete mathematikdidaktische Lehre. Dabei steht die Identifizierung und reflektierte Analyse vorhandener digitaler Ansätze und Tools und Planung, Vorbereitung, Durchführung und Nachbereitung der Lehrveranstaltungen im Vordergrund, wobei Förderliches und Hemmendes jeweils dokumentiert wird. Hierauf folgt die zweite methodische Phase, die systematisch empirisch geplant ist und in der neben der Umsetzbarkeit und Akzeptanz auch die Wirksamkeit untersucht werden soll (vgl. Ausblick). Aktuell ist die erste methodische Phase weit fortgeschritten. Ein erstes Ergebnis ist das MathEdu Digital-Lehrkonzept (vgl. unten), nach dem seit 2018 regelmässig an der PH Schwäbisch Gmünd und der Universität Ulm in den Masterstudiengängen für das Mathematiklehramt für die Sekundarstufen I und II gelehrt wird. Bisher haben 54 Studierende in 5 Kursen daran teilgenommen. Dabei wird kontinuierlich qualitativ untersucht, welche der in der Lehre angebotenen digitalen Tools von den Studierenden verwendet wurden und welche Erfahrungen die Studierenden damit im Rahmen ihrer Lernprozesse gemacht haben. Um die Sicht der Studierenden auf die 
eingesetzten Tools zu erfahren, werden folgende Erhebungsinstrumente eingesetzt, wobei den Studierenden ausdrücklich vermittelt wird, dass ihre Rückmeldungen für eine Verbesserung des Lehrkonzepts genutzt werden sollen:

- Austausch und persönliche Gespräche während und nach den Präsenzveranstaltungen

- Spontanbefragungen mit der App während der Präsenzveranstaltungen

- Online-Befragungen zum Abschluss der Webinare

- Grosser Abschlussfragebogen nach Beendigung des Semesters.

Die Befragungen mit der App und im Rahmen des Webinars bestehen in der Regel aus 4 bis 6 Fragen, die mit Hilfe von Multiple Choice zu beantworten sind, wobei es zum Teil mehrere Antwortmöglichkeiten gibt. Der grosse Abschlussfragebogen ist thematisch in die Fragenblöcke "Zur Nutzung der digitalen Materialien», "Zur Eignung der digitalen Materialien», «Zum Gesamtformat der Lehrveranstaltung», «Eigene Erfahrungen» gegliedert, und enthält abschliessend noch spezifische Fragen zu den fachlichen Inhalten und die Möglichkeit, Weiteres anzusprechen. Jeder Fragenblock ist in Unterabschnitte unterteilt, in denen jeweils spezifisch Fragen zu dem jeweiligen digitalen Tool zu beantworten sind. Dabei gibt es die Möglichkeit, Antworten anzukreuzen und gleichzeitig eigene Texte zu ergänzen. Immer wieder werden Studierende auch zur Konkretisierung durch eigene Texte aufgefordert, etwa durch vorgegebene Sätze, die zu ergänzen sind. Die Auswertung erfolgt qualitativ, indem die Antworten aus den Datensätzen der vier Befragungsmethoden jeweils Fragenkategorien (einzelne digitale Lehrelemente bzw. Lehrkonzept) zugeordnet und dann abgeglichen werden. So können auch Widersprüche aufgedeckt werden, die allerdings bisher nicht auftraten. Insgesamt interessieren in dieser ersten methodischen Phase sowohl die Mehrheitsmeinungen als auch die individuellen Aussagen einzelner Studierender.

\section{Das MathEdu Digital-Lehrkonzept}

Auf Basis der bisherigen Projektarbeit hat sich ein digitalisiertes Lehrkonzept herauskristallisiert, das das oben beschriebene Kriterium einer Mischung verschiedener Ansätze berücksichtigt und aus nicht-digitalen Elementen wie Präsenzveranstaltungen und digitalen Tools mit LOs wie VideoDemos (eigens erstellt oder aus Video Portalen), Apps, mobilen Technologien (Smartphones und Tablets), Aufgaben, Literatur bzw. Literaturquellen (pdf oder URLs zu Online-Dateien) besteht (vgl. Tabelle 2). Alles ist in einer Digital Library zusammengefasst und gespeichert und als Lehrformat werden immer wieder Webinare eingesetzt. Die Kursstruktur ist für alle Studierenden von Beginn an im LMS (hier Moodle) sichtbar und nachvollziehbar. Das Lehrkonzept entspricht in weiten Teilen dem Blended-Learning-Format, indem sich in Lernphase 
1 die Studierenden in die jeweilige Thematik selbstständig über VideoDemos, online bereitgestellte Literatur und zugehörige Aufgaben einarbeiten und sich dann in Lernphase 2 zusammen mit den anderen Studierenden und der Lehrperson in einer Präsenzveranstaltung, einem Webinar oder ggf. auch im LMS-Forum zum Austausch, u.a. auch in Kleingruppen, treffen. Gelegentlich werden physische Materialien, zum Beispiel zum Experimentieren usw., bereitgestellt, wie auch Tablets mit fachspezifischer Software. Während des Semesters besteht durchgehend die Möglichkeit zur Interaktion und Kommunikation, insbesondere über die Foren im LMS und über E-Mails. Gelegentlich wird ausdrücklich zum Austausch in den Foren aufgefordert, etwa indem einzelne Aufgabenbearbeitungen hochgeladen und diskutiert werden sollen. In den Präsenzveranstaltungen werden immer wieder spontan Befragungen über Apps eingesetzt. Der Auswahl der Lehrelemente ist eine intensive Recherche und Analyse im Hinblick auf die Verwertbarkeit für die spezifische mathematikdidaktische Lehre vorausgegangen. Da fast keine geeigneten Online-Kursangebote identifiziert wurden, wurden eigene zielgruppenspezifische Tools entwickelt und eingesetzt. Insgesamt hat sich eine eher «klassische» Blended-Learning-Kursstruktur herausgebildet, wenngleich die digitalen Formate in Lernphase 2 durchaus «innovativ» sind (Webinar und Forum). Das entwickelte Lehr-Format empfiehlt sich, da es einerseits auch für weniger digital erfahrene Lehrende eine unkomplizierte barrierearme Orientierung bietet und andererseits offen für eine schrittweise Weiterentwicklung ist. So können einzelne Lehrelemente jederzeit themen- und zielgruppenorientiert durch weitere fachliche Tools wie z.B. interaktive online-Experimente, Spiel-Apps, Lern-Tracker, Tutorials, Wikis oder Forschungsvideos zu Fallstudien ersetzt oder ergänzt werden. Insofern eignet sich das Lehrkonzept z.B. auch für die forschungsorientierte Lehre.

\begin{tabular}{|c|c|c|}
\hline & Lernphase 1 & Lernphase 2 \\
\hline Thema 1 & $\begin{array}{l}\text { Selbststudium (Pflicht): } \\
\text { - } \quad \text { VideoDemos } \\
\text { - Je nach Thema auch: Literatur (on- } \\
\text { line oder pdf) } \\
\text { - } \quad \text { Aufgaben (pdf) } \\
\text { Kommunikation und Interaktion: } \\
\text { - } \quad \text { Studierenden-Lerngruppen (online } \\
\text { oder face-to-face, optional) } \\
\text { - } \quad \text { Austausch und Feedback-On-De- } \\
\text { mand von Dozentin (durchgehend } \\
\text { möglich, z.B. per Email oder im Fo- } \\
\text { rum, optional) }\end{array}$ & $\begin{array}{l}\text { - Präsenzveranstaltungen an der } \\
\text { Universität mit Einsatz von power- } \\
\text { point-Präsentationen, sowie APPs an } \\
\text { Smartphones oder Tablets (Pflicht) } \\
\text { und/oder } \\
\text { - Webinar (virtuelles Meeting; Ort frei } \\
\text { wählbar) (Pflicht) } \\
\text { und/oder } \\
\text { - Diskussion im Forum (LMS) (gele- } \\
\text { gentlich Pflicht, z.B. nach dem Hoch- } \\
\text { laden von Lösungen im Studieren- } \\
\text { denordner (LMS)); hier: grundsätzli- } \\
\text { ches Feedback durch Dozentin }\end{array}$ \\
\hline Thema 2 & wie oben & wie oben \\
\hline usw. & & \\
\hline
\end{tabular}

Tab. 2.: Kursstruktur, angelehnt an das Blended-Learning-Format. 


\section{Ergebnisse}

Im Folgenden werden die Ergebnisse aus der ersten Projektphase, also aus den eigenen Lehrerfahrungen und den Befragungen und dem persönlichen Austausch mit den Studierenden, zusammengefasst. In dieser ersten Projektphase geht es zunächst um die Einordnung der digitalen Tools. Die Ergebnisse betreffen (weitgehend) einheitliche Rückmeldungen der Studierenden über alle Befragungsformate hinweg. Unterschiedliche Meinungen oder Einzelmeinungen werden aber stets auch beachtet und sind in der Zusammenfassung ausdrücklich gekennzeichnet. Aussagen über besondere Lernerfolge oder einen Mehrwert gegenüber rein analoger Lehre können an dieser Stelle nicht getroffen werden (vgl. Ausblick).

\section{Zur digitalisierten Lehre im Blended-Learning-Format}

Aus Lehrendenperspektive erscheint das Format des Blended-Learning mit Inverted bzw. Flipped Classroom-Anteilen sehr gut geeignet für die mathematikdidaktische Lehre in den Masterstudiengängen. Die Studierenden werden immer wieder zur Arbeit an den Themen gefordert, was durch die Lernplattform mit der Digital Library unterstützt wird (vgl. Abschnitt zu LMS). Auch in der Studierendenbewertung wurde der Wechsel zwischen VideoDemos und Präsenzveranstaltungen als geeignetes Lehrformat gelobt. Bezüglich der Besonderheit des MathEdu Digital-Lehrkonzepts, auch die Austauschphase (allgemein üblich als Präsenzphase) immer wieder digital zu gestalten, hat sich das Webinar als geeignet erwiesen, indem es hohe Anforderungen an die Interaktions- und Kommunikationsmöglichkeiten erfüllt (vgl. Abschnitt zu Webinar bzw. Interaktion).

\section{Zum Einsatz des LMS}

Das LMS (hier Moodle) stellt den Kern der Lehrveranstaltung dar. Schon vor Beginn des Semesters wird dort die Struktur der Lehrveranstaltung eingestellt, so dass sie für die Studierenden stets sichtbar und nachvollziehbar ist. Eventuelle Anpassungen erfolgen während der Vorlesungszeit. Die Lehrveranstaltung gliedert sich in 10 bis 11 Themenblöcke, wovon jeder die digitalen LOs für Lernphase 1 und eventuell Hinweise auf Quellen im Literatur- und Linkordner (auch in LMS) enthält, sowie einen Arbeitsauftrag mit Deadline und Angaben, wie sich Lernphase 2 gestaltet. Zum Teil sind im Forum Aufgabenbearbeitungen, z.B. zur Nutzung in Präsenzveranstaltungen, hochzuladen. Grundsätzlich sind die Studierenden dazu aufgefordert, den Wunsch nach einer (zusätzlichen) Präsenzveranstaltung mitzuteilen oder sie können sich selbst ein Format für Lernphase 2 wünschen. Zum Abschluss jeder Themeneinheit werden stets auch alle in Lernphase 2 eingesetzten Materialien, wie z.B. Präsentationen oder Aufgabenbearbeitungen usw., im LMS eingestellt. 
Ergebnisse: Der Einsatz des LMS wird insgesamt als sehr geeignet eingestuft. Aus Lehrendenperspektive unterstützt die Lernplattform mit der Digital Library das oben angesprochene Kriterium des «Knowledge-Pull». Denn für die Studierenden sind klar strukturierte Zeit-, Arbeitspläne und Materialien vorgegeben und sie sind durchgehend gefordert, ihre Ergebnisse für alle sichtbar hochzuladen und zu diskutieren. In der Studierendenbewertung wurde die im LMS gewählte Struktur gelobt und die angebotenen LOs als sehr passend bewertet. Sehr positiv hervorgehoben wurden die Foren, deren Nutzung allerdings vorwiegend erst eines Impulses der Lehrenden bedurfte. Einige Studierende gaben an, dass sie durch die Beiträge der anderen Studierenden im Forum förderliche Anregungen zu den Aufgaben erhalten haben, und dass sich das Forum sehr gut für die Kommunikation und Interaktion eigne. Soweit dies erfragt wurde, wurden für die Lernphase 2 bevorzugt digitale Lehrformate gewünscht. Bisher wurde zweimal der Wunsch nach einer weiteren Präsenzveranstaltung geäussert.

\section{Zum Einsatz der VideoDemos}

$\mathrm{Zu}$ jedem Themenblock gibt es ein bis vier zielgruppenorientierte eigens erstellte VideoDemos der Längen zwischen 7 und 13 Minuten (sowie 30 Minuten).

Ergebnisse: Ein wichtiges Ergebnis der Befragung und des Austauschs mit den Studierenden ist, dass VideoDemos sehr empfehlenswerte digitale Tools sind. Die bereit gestellten VideoDemos wurden von den Studierenden (weitgehend) vollständig genutzt und als geeignet eingestuft, sowohl zur Einführung, als auch für einen Überblick und zur spezifischen Vertiefung eines Themas. Diese Bewertung könnte dem Kriterium «Erfahrene Nützlichkeit» nach (Lai und Rishekesh Ulhas 2012) entsprechen. Die Studierenden schätzen die selbstständige Arbeit mit VideoDemos, allerdings sollten diese nach ihrer Bewertung nicht länger als 15 Minuten sein. Die in der Fachliteratur oft hervorgehobene Möglichkeit der Pause wurde von den Studierenden nicht als hilfreich angesehen. Dem häufig geäusserten Wunsch, parallel nur die Folien (ohne Audio) zur Verfügung zu haben, wurde inzwischen durch einen zusätzlichen Folienordner im LMS nachgekommen. Aus Lehrendensicht ist die Erstellung der VideoDemos unkompliziert. Ist die Lehre über Power-Point-Folien vorbereitet, wird nach (einmaliger) Installation eines am Laptop angeschlossenen Mikrophons und der Software die Präsentation an einem beliebigen Ort gehalten und aufgenommen. VideoDemos können sehr arbeitserleichternd sein, wenn sie in den nächsten Semestern erneut verwendet werden. 


\section{Zum Einsatz weiterer LOS}

Zum Lehrkonzept gehört es, dass neben den VideoDemos weitere LOs im LMS bereitgestellt werden. Je nach Thema finden sich in den Themenblöcken konkrete Link-Angaben zu Online-Lectures, zu Video-Portalen (insbesondere Youtube) und zu Online-Literatur bzw. Literatur im Pdf-Format.

Ergebnisse: Nach Angabe der Studierenden wurden alle angegebenen Youtube-Quellen angesehen, die Literatur wurde allerdings nur nach Aufforderung bzw. wenn die Aufgaben ohne diese Literatur nicht bearbeitbar waren, genutzt.

\section{Zum Einsatz der Webinare}

Webinare werden im MathEdu Digital-Lehrkonzept so eingesetzt, dass sie einzelne Präsenzveranstaltungen vollkommen ersetzen. Sie sind auf eine Stunde Dauer ausgerichtet und enthalten in der Regel Abschnitte mit kurzen Video-Audio-Folien-Präsentationen der Lehrperson, Aufgabenbearbeitung im Chat durch die Studierenden, Audio-Austausch zu einer speziellen Frage der Lehrperson, Arbeit in Kleingruppen in den (virtuellen) Gruppenräumen mit anschliessender Ergebnispräsentation am Whiteboard, sowie eine Abschlussbefragung. Zur Teilnahme an den Webinaren müssen sich die Studierenden anhand der bereit gestellten LOs vorbereiten. Für die Kommunikation und Interaktion während eines Webinars wurden bisher insbesondere die Chat- und die Audio-Funktion genutzt, sowie Video der Lehrperson, Folien und Whiteboard. Dabei wird jeweils unterschieden, ob die Nutzung im Hauptraum oder im Gruppenraum stattfindet. Im Hauptraum haben die Studierenden alle gemeinsam dieselbe Ansicht, während im Gruppenraum einzelne Studierende gemeinsam arbeiten, wobei sich die Lehrperson zuschalten kann oder nicht. Im Gruppenraum haben die Studierenden automatisch Audio- und Videoberechtigung, während diese im Hauptraum durch die Lehrperson spezifisch zugeteilt wird. Chatmöglichkeit besteht immer.

Ergebnisse: Das Webinar ist ein leistungsstarkes digitales Tool, das sich als eines von verschiedenen Lehrelementen für die mathematikdidaktische Lehre empfiehlt. Es enthält verschiedene Werkzeuge zur Interaktion und Live-Kommunikation, hat aber hinsichtlich einer intensiven Diskussion auch seine Grenzen. Für manche Themen ist es besser geeignet und es hat sich ein Wechsel mit Präsenzveranstaltungen bewährt, so dass nicht mehr als 3 Webinare pro Semester angeboten werden. Die Studierenden nutzen die Webinare gerne; mehrfach wurde auch der Wunsch geäussert, Präsenzveranstaltungen durch Webinare zu ersetzen (wobei einzelne Studierende sich auch eine weitere Präsenzveranstaltung wünschten). Im genaueren Austausch zeigte sich, dass der Lernerfolg ähnlich gut eingestuft wird, die Webinare aber komfortabler 
sind: man spart die Fahrt zur Universität und kann es sich zu Hause gemütlich machen. Dies entspricht dem Akzeptanzkriterium «Bequemlichkeit». Einige Studierende geben an, dass sie im Webinar eine gute Möglichkeit sehen, weitere digitale Erfahrungen zu sammeln. Interessant ist auch der Vorschlag einer Studentin, digitale Lehre und Präsenzveranstaltungen stärker zu differenzieren, indem letztere vorwiegend für eigenes Probieren (z.B. auch mit Materialien) genutzt werden sollten. Über die in Webinaren integrierten Funktionen wie Chat, Audio, Video, Gruppenraum usw. herrschen bei den Studierenden weitgehend einheitliche Meinungen (vgl. unten). Für die Lehrenden ist die Vorbereitung eines Webinars nicht kompliziert. Nach Installation der Anwendersoftware gelangt man über die entsprechende URL (hier: https://webconf.vc.dfn) schnell auf die entsprechende Seite, bei der ein Webinar durch Anklicken eines Buttons fast automatisch angelegt werden kann. Es müssen nur noch die entsprechenden (Präsentations-)Dateien hochgeladen werden. Für die Vorbereitung hat sich die im Projekt MathEdu Digital entwickelte tabellarische Vorlage bewährt (vgl. «Regieplan» unten und im Anhang).

\section{Zum Einsatz mobiler Technologien und Apps}

Eine regelmässige Komponente in den Präsenzveranstaltungen ist der Einsatz von Spontanbefragungen über eine entsprechende Voting-App (hier PINGO), sowie (seltener) die Nutzung von Tablets mit spezifischer Software zum Bearbeiten von Aufgaben (hier: schulisch relevante Mathematiksoftware). Für die Spontanbefragungen nutzen die Studierenden ihre eigenen Smartphones.

Ergebnisse: Die Spontanbefragungen wurden sehr gelobt und auch als motivierend bezeichnet (Aspekt «Erfahrene Freude»). Ebenso wird dem Einsatz der Tablets in der gewählten Form eine für das eigene Studium bereichernde Funktion zugeschrieben, wobei die Lehramtsstudierenden des Lehramts Sekundarstufe I lieber ihren eigenen Laptop als ein ausgeteiltes Tablet nutzen.

\section{Interaktion, Kommunikation und Zusammenarbeit zwischen Studierenden}

Ein besonderes Untersuchungsinteresse im Projekt MathEdu Digital richtet sich auf die Interaktion, die Kommunikation und die Zusammenarbeit zwischen den Studierenden (vgl. dazu auch Beckmann 2019b). Die bisherigen Erfahrungen und Rückmeldungen sprechen dafür, dass hierfür die Chatfunktion des Webinars und die Arbeit im Gruppenraum während eines Webinars als sehr förderlich und leistungsstark angesehen werden können, ebenso aber auch die persönlichen Treffen zwischen den Studierenden zur gemeinsamen Arbeit an den Aufgaben oder zur Vorbereitung einer Präsentation. Vorteile der Chat-Funktion sind, dass sie vielfältig einsetzbar ist und 
eine gleichzeitige Beteiligung aller Studierenden ermöglicht. Gegenüber einer Präsenzveranstaltung ist dies ein klarer Vorteil. Gemäss der Befragung schätzen die Studierenden die Chat-Möglichkeit und ihren spontanen störungsfreien Austausch einschliesslich der Möglichkeit, vorbereitete digitale Aufgabenlösungen schnell einfügen zu können. Sie halten die Chat-Interaktion auch für den mathematikdidaktischen Kompetenzerwerb für geeignet. Einzelne Studierende halten einen zusätzlichen Audio-Austausch für notwendig, da damit ein «schnellerer und genauerer Austausch» möglich sei. Allerdings gab es auch viele Vorbehalte gegenüber der Audio-Funktion, einerseits da die anderen Funktionen mehrheitlich als gewinnbringender eingestuft wurden, wohl aber auch aufgrund anfänglicher Hemmungen und technischer Unkenntnisse, die allerdings durch Vorbereitung in einem Test-Meetingraum ausgeglichen werden können. Unabhängig davon scheint die Präsenzveranstaltung beim Audio-Austausch gewisse Vorteile zu haben, da dieser spontaner und wenig geregelt erfolgen kann (z.B. entfällt das Freischalten des Mikrofons oder die Zuteilung von besonderen Rollen). Ebenso könnte das Fehlen von kontinuierlichen verbalen und non-verbalen Signalen und Regungen aus der ganzen Gruppe im Webinar, wie sie in Präsenzveranstaltungen empfangen werden, dazu führen, dass nicht immer alle Studierenden angemessen genug einbezogen und an der Interaktion beteiligt werden. So gaben einzelne Studierende in einer Webinar-Abschlussbefragung an, dass sie sich gerne noch mehr beteiligt hätten. Die im Webinar integrierten Icons wie zum Beispiel «Melden» wurden von den Studierenden bisher wenig genutzt. Für die Kommunikation und Interaktion erscheint auch der Gruppenraum besonders interessant. Während Studierende im Hauptraum auf ihr Live-Video und die Moderatorrolle mit erweiterten Funktionen wie Audio-Präsentationsmöglichkeiten gerne verzichteten, hatten sie im Gruppenraum keine Probleme damit. Im Gruppenraum wurden intensive Diskussionen - auch unter Nutzung des Whiteboards für Ideenskizzen - beobachtet, wobei die Interaktionsdynamik aus den Gruppenräumen in den Hauptraum übertragen wurde. Die Studierenden schätzen den Austausch mit anderen Studierenden in der Kleingruppe und sie stufen die anschliessende Präsentation förderlich für die Kommunikation und Besprechung der Aufgaben ein. Auch dass dieses Format für viele Studierende etwas ganz Neues und Produktives in der universitären Lehre darstellte, machte die Arbeit im Gruppenraum für viele interessant.

\section{Motivation und Material für Lehrende}

Um dem Ziel gerecht zu werden, Lehrende auch mit weniger Erfahrung oder Affinität zu digitaler Lehre dafür zu interessieren, werden im Projekt MathEdu Digital neben den Möglichkeiten der Digitalisierung, zunächst erfahrungsbasiert, die Barrieren (u.a. auch Zugangsvoraussetzungen und Aufwand für Lehrende), Chancen und Gelingensbedingungen identifiziert und ein Unterstützungstool für Lehrende entwickelt. 
Im Ergebnis soll den Lehrenden ein Paket zur Verfügung stehen, mit dem sie unkompliziert und unter Berücksichtigung der unterschiedlichen digitalen Lehrelemente und Lernaktivitäten der Studierenden die verschiedenen digitalen Möglichkeiten umsetzen können. Die Einstiegsbarrieren sollen dabei möglichst klein gehalten werden und es sollen konkrete praktische Hinweise und direkt einsetzbare Materialien enthalten sein. Bisher konnten bereits erfahrungsbasierte Ergebnisse bezüglich der Art der Materialien gewonnen und erste Elemente für das MathEdu Digital-Digitalisierungspaket entwickelt werden. Dazu gehört insbesondere auch der «Regieplan», d.h. eine Tabelle für die Durchführung eines Webinars, die direkt ausgefüllt und somit an die Bedürfnisse des eigenen Webinars angepasst werden kann (vgl. Anhang).

\section{Ausblick}

Die qualitativen und erfahrungsbezogenen Ergebnisse deuten auf eine Eignung des MathEdu Digital-Lehrkonzepts mit den benutzten Lehrelementen wie insbesondere LMS, VideoDemos, Webinaren und Apps als Grundkonzept für die digitale Lehre hin. Dieses Grundkonzept ist inzwischen weitgehend ausgereift, so dass es 2020 in die «Routine» und ab 2021 in die systematische Erprobungsphase gehen kann. Dabei sollen neben der Akzeptanz dann auch die Lernwirksamkeit empirisch untersucht werden, wobei sowohl fachlicher als auch digitaler Kompetenzerwerb erfasst werden sollen. Die Entscheidung über die Forschungsmethoden, zum Beispiel unter Berücksichtigung von Learning Analytics, Educational Data Mining usw. wird parallel zur letzten Ausreifung des Lehrkonzepts erfolgen. Für die Erfassung der fachlichen Kompetenz bieten sich vermutlich eher themenbezogene (abschnittsweise) angelegte Tests und Befragungen an, die den absoluten Lernerfolg erfassen. Da das Lehrkonzept spezifisch für die 2018 neu eingeführten Lehramtsmasterstudiengänge in Baden-Württemberg entwickelt wurde, stehen vergleichbare rein nicht-digitale Lehrkonzepte nicht zur Verfügung. Vergleichende Untersuchungen bieten sich allerdings vor dem Hintergrund der «Perspektive der Medien» (vgl. oben) für die Erfassung der gewonnenen digitalen Erfahrungen der Studierenden an. Insgesamt steht die Fertigstellung der drei Projektbausteine, also (1) Inhalte und Medien, (2) Digitalisierte Lehre und (3) Digitalisierungspaket, an (vgl. oben), wobei auch beobachtet werden soll, inwieweit die erarbeiteten (möglichst barrierefreien) Materialien für die Lehrenden hilfreich sind und inwieweit diese das Lehrkonzept auch zur eigenen innovativen Weitentwicklung nutzen. 


\section{Literatur}

Borba , Marcelo, Petek Askar, Johann Engelbrecht, George Gadanidis, Salvador Llinares, und Mario Sánchez Aguilar. 2016. "Blended learning, e-learning and mobile learning in mathematics education». ZDM Mathematics Education 48: 589-610. https://doi.org/10.1007/ s11858-016-0798-4.

Beckmann, Astrid. 2019a. «MathEdu Digital - project overview and reflection on special aspects of digitization in mathematics teacher education». INTED 2019, 1842-1846. https:// doi.org/10.21125/inted2019.

Beckmann, Astrid. 2019b. «The Webinar as a digital tool in mathematics teacher education». INTED 2019, 1821-1825. https://doi.org/10.21125/inted2019.

Beckmann, Astrid. 2020. «Students views on digital tools in university lectures». INTED 2020 (in press). https://doi.org/10.21125/inted2020.

Chatti, Mohamed Amine, Mohammad Ridwan Agustiawan, Matthias Jarke, und Marcus Specht. 2010. «Toward a personal learning environment framework». International Journal of Virtual and Personal Learning Environments I (4), 66-85. https://doi.org/10.4018/jvple.2010100105.

Clements, Kati, Jan Pawlowski, und Nikos Manouselis. 2015. «Open educational resources repositories literature review - Towards a comprehensive quality approaches framework». Computers in Human Behaviour 51 (B): 1098-1106. https://doi.org/10.1016/j.chb.2015.03.026.

Cretchley, Patricia. 2005. «Mathematics and dumping lectures? Another perspective on the shift towards learner pragmatism». In Proceedings of the 5th South. Hem. Conf. On undergraduate mathematics and statistics teaching and learning 42-48. Queensland Australia,

Dehne, Julian, Ulrike Lucke, und Mandy Schiefner-Rohs. 2017. «Digitale Medien und forschungsorientiertes Lehren und Lernen - empirische Einblicke in Projekte und Lehrkonzepte». In Bildungsräume. Proceedings der 25. Jahrestagung der Gesellschaft für Medien in der Wissenschaft, 71-83. Chemnitz, Münster, New York: Waxmann.

Downes, Stephen.2005.«E-Learning2.0»e-learn2005(10). https://doi.org/10.1145/1104966.1104968.

Feinstein, Joel. 2010. «Using IT when teaching mathematics classes». Presentation at Higher Education Academy: Maths, Stats and OR Network Nottingham University. Nottingham UK.

Frerich, Sulamith, und Silke Frye. 2016. «Erfolgreiches Praxisbeispiel: Ein interaktives Blended-Learning-Seminar mit Praxisphase». In Teaching Trends 2016: Digitalisierung in der Hochschule: Mehr Vielfalt in der Lehre, hrsg. v. Wolfgang Pfau, Caroline Baetge, Svenja Mareike Bedenlier, Carina Kramer u. Joachim Stöter, 199-208. Münster, New York: Waxmann. ISBN 978-3-8309-3548-3. http://d-nb.info/1116096781.

Gadanidis, George, und Marcelo Borba. 2008. «Our lives as performance mathematicians». For the Learning of Mathematics 28 (1): 44-51. http://www.rc.unesp.br/downloads/artigos/gadanidis_borba/gadanidis_borba_our_lives_as_performance_mathematics.pdf.

Gadanidis, George. 2012. «Why can 't I be a mathematician?». For the Learning of Mathematics" 32 (2): 20-26. 
Gegenfurter, Andreas, Karina Fisch, und Martina Reitmaier-Krebs. 2017. «Disziplinäre Fachkultur als Einflussgröße auf die studentische Akzeptanz von E-Learning-Angeboten an Hochschulen». In Jahrestagung der Gesellschaft für Hochschulforschung: Digitalisierung der Hochschulen: Forschung, Lehre, Administration, hrsg. von DZHW, 6-7.Hannover. https:// www.gfhf.net/assets/2016/07/Abstractband_GfHf2017.pdf\#page=12.

Guo, Yan Ru, Dion Goh, und Brendan Luyt. 2017. «Tertiary students' acceptance of a game to teach information literacy». Aslib Journal of Information Management 69 (1): 46-63. https:// doi.org/10.118/AJIM-08-2016-0131.

Hahn, Heike, und Nadine Puschner. 2017. «Von der Präsenzvorlesung zu 'Mini-Lectures' - Umsetzung eines Inverted Classroom Szenarios». BzMU 2017: 377-380. Münster: WTM. https:// eldorado.tu-dortmund.de/bitstream/2003/36509/1/BzMU-2017-HAHN.pdf.

Hetzner, Sónia, Claudia Schmidt, Katja Sesselmann, und Stefanie Zepf. 2017. «Pimp your lecture: Erfolgreiche Ansätze zur Unterstützung der Digitalisierung der Lehre an der FriedrichAlexander-Universität Erlangen-Nürnberg». In Bildungsräume. Proceedings der 25. Jahrestagung der Gesellschaft für Medien in der Wissenschaft, hrsg. v. Christoph Igel, 110-115. Chemnitz, Münster, New York: Waxmann.

Jokiaho, Annika, und Birgit May. 2017. «Hindernisse für die Nutzung von E-Learning an Hochschulen: Aktueller Forschungsstand». In Bildungsräume. Proceedings der 25. Jahrestagung der Gesellschaft für Medien in der Wissenschaft, hrsg. v. Christoph Igel, 20-31. Chemnitz, Münster, New York: Waxmann.

Lai, Jung-Yu, und Khire Rushikesh Ulhas. 2012. "Understanding acceptance of dedicated etextbook applications for learning». The Electronic Library 30 (3): 321-338. https://doi. org/10.1108/02640471211241618.

Le, Ada, Steve Joordens, Sophie Chrysostomou, und Raymund Grinnell. 2010. "Online lecture accessibility and its influence on performance in skills-based courses». Comput. Educ. 55: 313-319. https://doi.org/10.1016/j.compedu.2010.01.017.

Makori, Elisha Ondieki. 2015. «Micro factors influencing use of electronic information resources among postgraduate students in institutions of higher learning in Kenya». Library Hi Tech News 32 (1): 18-21. https://doi.org/10.1108/LHTN-10-2014-0096.

Mayrberger, Kerstin. 2017. «Student Engagement und Digitalisierung - Erfolgsfaktoren für eine zeitgemäße Lehre und Administration an der Hochschule». In Jahrestagung der Gesellschaft für Hochschulforschung: Digitalisierung der Hochschulen: Forschung, Lehre, Administration, hrsg. v. DZHW, 20-21. Hannover. https://www.dzhw.eu/gfhf2017/pdf/Abstractband_GfHf2017.pdf\#page=26.

Rettig, Lars, und Tim Warszta. 2016. «Der Einfluss von Kursdesignelementen auf Studierendenzufriedenheit und Studierendenloyalität». In Teaching Trends 2016. Digitalisierung in der Hochschullehre: Mehr Vielfalt in der Lehre, hrsg. v. Wolfgang Pfau, Caroline Baetge, Svenja Mareike Bedenlier, Carina Kramer u. Joachim Stöter, 177-190. Münster, New York: Waxmann. ISBN 978-3-8309-3548-3. http://d-nb.info/1116096781.

Rickard, Wendy. 2010. The Efficacy (and Inevitability) of Online Learning in Higher Education. Boston: Pearson Learning Solutions. www. pearsonlearningsolutions.com 
Owston, Ron, Denys Lupshenyuk, und Herb Wideman. 2011. «Lecture Capture in Large Undergraduate Classes: What is the Impact on the Teaching and Learning Environment?». In Annual Meeting of the American Educational Research Association. New Orleans LA USA. https://www.researchgate.net/publication/228728272.

Sailer, Michael, Florian Schutz-Pernice, Olga Chernikova, Maximilian Sailer, und Frank Fischer. 2018. Digitale Bildung an bayerischen Hochschulen - Ausstattung, Strategie, Qualifizierung und Medieneinsatz, hrsg. v. vbw. München. https://www.vbw-bayern.de/Redaktion/ Frei-zugaengliche-Medien/Abteilungen-GS/Bildung/2018/Downloads/FINAL_Digitale_Bildung_an_bayerischen_Hochschulen_2.pdf.

Söbke, Heinrich, und Maria Reichelt. 2016. "'Rat(en) in der Lehre` - Über die Spiel(un)lust unserer Studierender am Beispiel digitaler Apps». In Teaching Trends 2016. Digitalisierung in der Hochschule: Mehr Vielfalt in der Lehre, hrsg. v. Wolfgang Pfau, Caroline Baetge, Svenja Mareike Bedenlier, Carina Kramer u. Joachim Stöter, 163-175. Münster, New York: Waxmann. http://d-nb.info/1116096781.

Trenholm, Sven, Lara Alcock, und Carol L. Robinson. 2012 und 2015 (Ergänzung). «Mathematics lecturing in the digital age». Int. J. Math. Educ. Sci. Technol 43 (6): 703-716 und 46 (3): 480. https://doi.org/10.1080/0020739X.2011.646325.

Venkatesh, Viswanath, Michael Morris, Gordon Davis, und Fred Davis. 2003. «User Acceptance of Information Technology: Toward a Unified View». MIS Quarterly 27 (3): 425-478. https:// doi.org/10.2307/30036540.

Venkatesh, Viswanath, und Xiaojun Zhang. 2010. «Unified Theory of Acceptance and Use of Technology: U.S. Vs. China». Journal for Global Information Technology Management 13 (1): 5-27. https://doi.org/10.1080/1097198X.2010.10856507.

Winteler, Adi, und Peter Forster. 2008. «Lern-Engagement der Studierenden. Indikator für die Qualität und Effektivität von Lehre und Studium». Das Hochschulwesen 56 (6): 162-170. http://www.hochschulwesen.info/inhalte/hsw-6-2008.pdf. 
Anhang: Muster für einen «Regieplan» für die Durchführung eines Webinars

Hinweise zur Nutzung: Der Regieplan dient zur Planung des eigenen Webinars und stellt eine prinzipielle Struktur dar, die sehr einfach individuell angepasst werden kann. Zum Beispiel können die Titel der PowerPoint-Präsentationen oder der Aufgaben eingefügt oder gelöscht und weitere persönliche Zusatzhinweise zur technischen Handhabung der Plattform in der linken Spalte ergänzt werden usw. Grüne Markierungen bedeuten: hier muss ich als Lehrperson aktiv sein. Gelbe Markierungen bedeuten: hier müssen die Studierenden aktiv sein. Bei den grauen Markierungen sind noch die Webinardaten einzutragen.

\begin{tabular}{|c|c|c|}
\hline \multicolumn{3}{|c|}{ Zugangscode: XXXXXXXXXXX } \\
\hline $\begin{array}{l}\text { Zeit und Zusatz- } \\
\text { hinweise }\end{array}$ & $\begin{array}{l}\text { Dozentin, } \\
\text { Inhalt und Methode }\end{array}$ & $\begin{array}{l}\text { Studierenden-aktivität } \\
\text { und Methode }\end{array}$ \\
\hline \multicolumn{3}{|c|}{$\begin{array}{l}\text { Webcam und Mikrophon verbinden und einschalten (Symbole: grün!) } \\
\text { Begrüssungs-PowerPoint einstellen }\end{array}$} \\
\hline \multirow[t]{5}{*}{$X X \cup h r$} & Begrüssung (in Kamera schauen!) & Im Chat «Hallo» sagen \\
\hline & $\begin{array}{l}\text { Vorbereitung, Mikrophon- und Bild-Test, } \\
\text { Hinweise zu «Melden» } \\
\rightarrow \text { Chat, Symbole }\end{array}$ & $\begin{array}{l}\text { Durch Studierende } \\
\text { bestätigen lassen, dass } \\
\text { sie mich sehen und } \\
\text { hören }\end{array}$ \\
\hline & Ppt 1: Einstieg Übersicht zu Thema1 & \\
\hline & 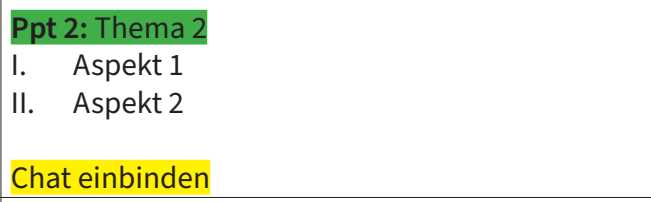 & $\begin{array}{l}\text { Studierende tragen im } \\
\text { Chat ein }\end{array}$ \\
\hline & $\begin{array}{l}\text { Ppt 3: Thema } 3 \\
\text { Eigene Präsentation, } \\
\text { Vorstellung der Aufgabe }\end{array}$ & \\
\hline \multirow[t]{3}{*}{$\begin{array}{l}10 \text { Minuten? } \\
\text { X Arbeitsgruppen } \\
\begin{array}{l}\text { Automatisgruppen starten } \\
\text { teilen }\end{array}\end{array}$} & $\begin{array}{l}\text { Aufgabe (vgl. Ppt 3) } \\
\text { Teilnehmerinnen in Gruppenräume ziehen bzw. } \\
\text { automatisch verteilen. } \\
\text { Nachricht in Arbeitsgruppen senden } \\
\text { Arbeitsgruppensitzung beenden, } \\
\text { Teilnehmerinnen auf Moderator schalten } \\
\text { Pods > Arbeitsgruppen-Pods > Whiteboards }\end{array}$ & $\begin{array}{l}\text { Studierende bearbei- } \\
\text { ten die Aufgabe } \\
\text { in Gruppen } \\
\text { Einzelne Studierende } \\
\text { stellen AG-Ergebnis vor }\end{array}$ \\
\hline & \multicolumn{2}{|l|}{ Zum Abschluss: Abstimmung: 6 Fragen } \\
\hline & Verabschiedung, ggf. Screenshot & \\
\hline
\end{tabular}

